\title{
Changes in sight
}

Starting its work ahead of the Brazilian Society of Dermatoloy, the new editorial board intends to implement some changes aiming mainly at improving the quality of the journal.

A decision considered crucial is the standardization of the print magazine exclusively in Portuguese, since this version is directed primarily to domestic readers. Thus, the national authors and foreigners who have Portuguese as their native language must submit their work in Portuguese, being given them the option to attach a translation of the article in English. The studies of foreign authors of other languages should be submitted in English; these studies, however, will be translated into Portuguese, wich will be afforded by the magazine, to be published in the printed journal. Thus, the printed magazine will have a uniform standard in a single language, attending the interests of all national readers, including even who does not master reading in English. However, the online version of the journal will remain in English, attending the interest of foreign readers and ensuring international visibility for the journal.

Another change will be the return of the section "What's your diagnosis", considering the importance of image in the formulation of the diagnosis in this specialty. This section is intended, therefore, to provide a brief report of a case of undeniable interest, illustrated by clinical imaging, and usually also by complementary examination, especially histopathology, in which the final diagnosis of the clinical presentation is questioned, followed by a brief discussion of the disease.

It is still important to highlight the limitation of the number of authors according to the nature of the article. Based on strict criteria of authorship contained in the guidelines of the journal, the maximum number of four authors was considered sufficient for the different sections of the journal, except for investigation and review articles, which will be limited to six authors. However, exceptionally, editors may admit a larger number of authors, when the complexity of the study justifies it.

With implications in the impact factor, dependent on citations of articles published in the magazine, it becomes forcible to raise the rigor in the selection criteria of the articles, especially clinical cases, for which editors will be more attentive regarding quality. Thus, reports of cases considered simpler should be directed to the section "Letter to the Editor", which will have more limited space for text, illustrations and references.

Finally, in order to speed the publication process and make less burdensome the task of the reviewers, the new editors appeal to the authors to put great care in the preparation of their manuscripts, with close attention to the correct use of the language, to the publishing standards of the journal and to the technical quality of the illustrations. 